\title{
Les jeunes auteures francophones de bandes dessinées à Montréal : pratiques, réseaux, représentations
}

\author{
Anna Giaufret \\ Università di Genova
}

Notre contribution se penchera sur la production des femmes appartenant à la communauté des jeunes auteurs montréalais (nés après 1980), sans oublier leur apport théorique (Delporte) ${ }^{1}$. Nous commencerons par tracer un panorama quantitatif de la présence féminine dans la jeune bande dessinée montréalaise (maisons d'éditions, revues, réseaux, médias, etc.), pour ensuite regarder de plus près les pratiques éditoriales et artistiques de ces auteures (collaborations, genres, etc.) afin d'identifier la diversité de l'apport féminin à la bande dessinée. Nous allons par exemple avancer l'hypothèse que la présence féminine est très forte dans le milieu de la bande dessinée indépendante, mais beaucoup moins dans la production mainstream et que, contrairement à d'autres pays, elle n'est pas dominante dans les blogues.

\section{Introduction : l'essor de la $\mathrm{BD}$ au Québec à partir de 2000}

Si l'on en croit le dernier bilan tracé de la bande dessinée au Québec, le Bilan BDQ 2014 de Michel Viau (Viau 2014), la BD québécoise connaît un moment d'essor: Viau mentionne en effet une augmentation des publications de bandes dessinées au Québec de $57 \%$ par rapport à 2009 et de $342 \%$ par rapport à 1999. Sur un total de 168 albums publiés en 2014, $131(81,5 \%)$ sont en français et 37 en anglais $(18,5 \%)$. Si l'on tient compte du fait que seulement 55 des BD en français sont des traductions, on obtient le chiffre de 76 albums originaux publiés en français au Québec, c'est-à-dire la majorité. Deux des cinq premières maisons d'édition ${ }^{2}$ en termes d'albums BD publiés en 2014 sont à Montréal : La Pastèque et Pow Pow, qui sont responsables de 7,3\% (10 albums) et 4,4\% (6 albums) des publications en langue française, toutes originales. Outre ces deux maisons, Mécanique générale, La Mauvaise tête/Colosse et la revue Planches ont aussi leur siège à Montréal. Du côté anglophone, Drawn \& Quartely domine largement avec $71 \%$ de la production en langue anglaise au Québec. C'est donc vers ces

\footnotetext{
1 Nous tenons à remercier ici tous les auteurs mentionnés dans cet article, tout particulièrement Sophie Bédard, Iris, Sophie Yanow et Zviane qui ont permis la reproduction de leurs travaux. Sans leur contribution, cet article n'aurait jamais vu le jour.

${ }^{2}$ Le bilan de Viau comptabilise aussi la maison Scholastic Canada (classée deuxième en termes de nombre de publications) qui a son siège à Toronto, puisqu'elle adresse une partie de ses publications essentiellement au marché québécois. Toutefois, cet élément fausse les données sur l'édition de la BD québécoise. La première maison d'édition est Presse Aventure (BD jeunesse), la quatrième Michel Quintin, qui publie des BD d'« aventure » pour le public adulte ainsi que des BD pour la jeunesse.
} 
maisons d'édition que nous allons nous tourner, afin de vérifier la présence d'auteures du point de vue quantitatif dans leurs catalogues, avant de passer aux pratiques de la $\mathrm{BD}$ au féminin, toujours dans le contexte montréalais. Nous ne tiendrons compte que des bandes dessinées publiées après 2000, et dans les faits pour la plupart publiées à partir de $2010^{3}$. Voyons d'abord comment se positionnent les femmes auteurs de BD dans le panorama éditorial montréalais.

\section{Présence féminine dans les maisons d'édition montréalaises}

La Pastèque, qui publie les aventures de Paul, de Michel Rabagliati, compte 75 albums dans son catalogue $\mathrm{BD}$, dont certains relèvent toutefois plutôt du genre «livre illustré ». Sur un total de 49 auteurs de bandes dessinées (d'après le classement de leur propre site), les femmes (scénaristes et dessinatrices) sont six (Isabelle Arsenault, Fanny Britt, Iris, Cathon, Violaine Leroy, Zviane) pour un total de six albums, parfois en collaboration entre elles (Arsenault et Britt, Cathon et Iris) ou avec d'autres auteurs (Iris et Yves Pelletier). Les auteures ne représentent donc ici que $12 \%$ du total, alors que leurs BD équivalent à $8 \%$. Seul l'album d'Arsenault et Britt s'adresse à un jeune public. Violaine Leroy est la seule auteure non québécoise.

Chez Pow Pow les rapports de genre sont différents : sur les 23 BD du catalogue, 9 (39\%) sont réalisées totalement ou en partie par des femmes, qui représentent 3 auteurs (Cathon, Iris et Zviane) sur 15 (20\%). À elles seules, Zviane et Sophie Bédard totalisent quatre bandes dessinées chacune, et sont ainsi, avec Pierre Bouchard et Francis Desharnais (auteurs des trois tomes de Motel Galactic ainsi que d'ouvrages réalisés seuls), les noms les plus fréquents dans le catalogue de Pow Pow.

La Mauvaise tête est la collection commerciale de la structure éditoriale Colosse, entièrement spécialisée en bandes dessinées et qui réalise des publications indépendantes sans distribution en librairie. La Mauvaise tête a donc un catalogue limité : elle signale sur son site 10 titres de bandes dessinées dont 3 sont réalisés par des femmes (30\%), et 9 auteurs dont trois femmes $(33 \%)$ : Ariane Dénommé, Pascaline Lefebvre, Sophie Yanow. Pour sa part, Colosse publie 44 auteurs dont 17 femmes (38\%).

Pour finir, Mécanique générale est une maison d'édition spécialisée en bandes dessinées et fondée par Jimmy Beaulieu. Même si d'après le rapport Viau elle arrive derrière La Pastèque et Pow Pow en 2014 avec quatre albums publiés, son riche catalogue compte 41 auteurs dont 6 femmes $(14,6 \%)$ : Bach, Catherine Lepage, Catherine Ocelot, Iris, Lolita Séchan, Zviane. Du

${ }^{3}$ Si l'on regarde le développement historique de la bande dessinée québécoise, il est possible de remarquer que Falardeau (Histoire) cite, dans l'Index des noms, 25 femmes sur un total de 258 noms. 
côté anglophone, Drawn and Quarterly ${ }^{4}$ est une grande maison d'édition qui jouit d'un prestige important, non seulement au Québec, mais au Canada et en Amérique du Nord. Elle affiche 86 auteurs sur son site, dont 24 femmes (27\% du total) qu'il serait ici trop long de nommer.

Il semblerait donc que, à part La Pastèque, qui n'est pas une maison d'édition spécialisée en bande dessinée, mais qui publie aussi des livres illustrés et des livres pour la jeunesse, et Mécanique générale, le pourcentage d'auteures se situe entre 20 et $33 \%$ sur l'ensemble des auteurs, ce qui constitue un résultat remarquable si on le compare aux données de l'ACBD de 2014 pour l'Europe francophone (Ratier 30) ${ }^{5}$. Cette différence relève-telle d'une plus grande ouverture au médium de la BD en Amérique du Nord? Ou bien plutôt aux choix stylistiques des maisons d'édition montréalaises ${ }^{6}$ qui ont exclu les albums d'aventure et se sont davantage tournés vers un style intimiste, autofictif, plus proche du roman graphique ou de la bande dessinée d'auteur que de la BD grand public? Nous sommes tentées de répondre par l'affirmative aux deux questions. Un troisième facteur joue probablement aussi : les femmes sont plus nombreuses parmi les jeunes auteurs. Or, les auteurs de ces maisons d'édition sont en grande partie nés après 1970, voire 1980.

\section{Du côté des revues}

Pour notre étude quantitative, nous allons aussi prendre en compte deux publications en série qui paraissent particulièrement significatives : la revue Planches et le magazine des 48 heures de la bande dessinée de Montréal.

Celui-ci a connu seulement trois numéros, correspondant aux trois éditions de la manifestation qui s'est produite en 2009, 2010 et 2011. Voici un schéma résumant les données quantitatives :

\begin{tabular}{|l|l|l|l|l|}
\hline Année & $\begin{array}{l}\text { Auteurs } \\
\text { total }\end{array}$ & $\begin{array}{l}\text { Auteurs } \\
\text { femmes }\end{array}$ & $\begin{array}{l}\mathbf{\%} \text { des } \\
\text { femmes }\end{array}$ & Directeur \\
\hline 2009 & 24 & 11 & $45 \%$ & $\begin{array}{l}\text { Julie Delporte } \\
\text { et Vincent } \\
\text { Giard }\end{array}$ \\
\hline
\end{tabular}

\footnotetext{
${ }^{4}$ Qui, ainsi que le souligne Saint-Jacques (82) est dirigée aujourd'hui par une femme, Peggy Burns.

${ }^{5}$ La récente « Charte du collectif des créatrices de bande dessinée contre le sexisme » signée par une centaine d'auteures « majoritairement françaises et belges, mais également italiennes, canadiennes, américaines... », dénonce « les aspects du sexisme dans l'industrie littéraire où nous évoluons, tout en énonçant des méthodes pour le combattre ». Ce collectif montre l'importance du problème, mais aussi une forte prise de conscience et de position.

${ }^{6}$ Nous n'avons pas analysé ici les maisons d'éditions qui ne consacrent qu'une moindre partie de leurs catalogues à la $\mathrm{BD}$, telles que L'Oie de Cravan qui publie par exemple Diane Obomsawin et Julie Doucet.
} 


\begin{tabular}{|l|l|l|l|l|}
\hline 2010 & 28 & 13 & $46 \%$ & $\begin{array}{l}\text { Julie Delporte } \\
\text { et Vincent } \\
\text { Giard }\end{array}$ \\
\hline 2011 & 26 & 10 & $38 \%$ & Vincent Giard \\
\hline
\end{tabular}

La présence féminine est ici très importante, même du point de vue éditorial. Sa légère baisse, après l'augmentation de 2010, coïncide d'ailleurs avec la disparition de Julie Delporte à la tête du magazine. La revue Planches, dirigée par Emilie Dagenais et Sandra Vilder propose sur son site une description de ses objectifs : Premièrement, il veut faire la promotion des auteurs de bande dessinée du Québec. Cette définition implique toute personne faisant partie intégrante du patrimoine québécois de la bande dessinée ou de tout autre métier s'y rapportant (illustrateur, encreur, coloriste, lettreur, scénariste, etc.). Deuxièmement, cette promotion se fait non seulement à travers la commande, la rémunération et la diffusion de leurs œuvres dans la revue imprimée trimestriellement, mais également via les articles et vidéos du site internet qui ont pour objectif de mettre de l'avant une discussion sur la bande dessinée. Le but est de valoriser un discours critique sur la production québécoise en l'analysant par toutes les approches possibles.

Intéressant et imposant défi que nous posons à la communauté des amateurs de bande dessinée québécoise, certes, mais nous avons confiance qu'ils seront nombreux à le relever! Au fil des mois, le site internet se peuplera de textes qui, nous l'espérons, appelleront à la réflexion et à l'interaction dans les commentaires. La revue montre aussi une sensibilité certaine pour les sujets tels que la sexualité (chronique de Sophie Bédard) et le féminisme (chronique de Bach à partir du numéro 3) comme en témoignent les chiffres suivants:

\begin{tabular}{|l|l|l|l|l|}
\hline Numéro & Année & $\begin{array}{l}\text { Total } \\
\text { auteurs }\end{array}$ & $\begin{array}{l}\text { Auteurs } \\
\text { femmes }\end{array}$ & \% femmes \\
\hline 1 & 2014 & 25 & 8 & $32 \%$ \\
\hline 2 & 2015 & 22 & 7 & $31 \%$ \\
\hline 3 & 2015 & 25 & 9 & $36 \%$ \\
\hline 4 & 2015 & 20 & 6 & $30 \%$ \\
\hline
\end{tabular}

Les auteures occupent donc une place encore plus importante dans ces deux publications. Le magazine des $48 \mathrm{~h}$ a malheureusement cessé de paraitre depuis longtemps, mais Planches semble assurer une relève intéressante dans le panorama de la BD québécoise, afin de promouvoir les nouveaux talents, avec une place de choix pour les auteures.

\section{Les réseaux et les médias}

Afin de tracer un panorama le plus possible complet de la BD à Montréal, il ne faut pas oublier le lieu qui regroupe les ateliers de nombreux auteurs ainsi 
que les sièges de quelques maisons d'édition: la Maison de la BD de Montréal. Son site classe les auteurs en trois catégories, dont voici les données :

\begin{tabular}{|l|l|l|l|}
\hline Catégorie & Auteurs total & $\begin{array}{l}\text { Auteurs } \\
\text { femmes }\end{array}$ & \% femmes \\
\hline Auteurs & 19 & 7 & $36 \%$ \\
\hline Auteurs invités & 34 & 19 & $55 \%$ \\
\hline Anciens & 39 & 20 & $51 \%$ \\
\hline
\end{tabular}

Ici, les femmes sont donc parfois la majorité, notamment dans les catégories « invités » et « anciens », mais elles représentent quand même plus d'un tiers des auteurs, qui regroupe aussi des individus qui ne sont pas des créateurs, mais des éditeurs, comme Emilie Dagenais et Sandra Vilder.

Quant aux émissions radio consacrées à la $\mathrm{BD}$, "Case départ» (CISM 89,3) a été animée jusqu'à sa fermeture par Amélie Mathieu, alors que Julie Delporte continue à animer avec deux collègues sur CHOQ l'émission «Dans ta bulle ». Moins féminine, la chaîne télé ARTV de Radio-Canada consacre de son côté une émission à la BDQC disponible en ligne et présentée par Sophie Cadieu : sur les 10 auteurs choisis, deux seulement sont des femmes (Zviane et Julie Rocheleau, toutes les deux montréalaises).

Il semblerait donc que la présence féminine soit très importante dans les circuits indépendants (en tant qu'auteures et éditrices), mais que l'élément féminin s'estompe dès qu'on s'approche des instances mainstream. Par ailleurs, il faut souligner que Montréal occupe une place centrale pour les jeunes auteurs qui s'y concentrent de plus en plus en y arrivant de l'Outaouais (Iris, mais aussi Fontaine Rousseau et Samson-Dunlop), du Lac Saint-Jean (Pascal Girard), mais aussi des États-Unis (Sophie Yanow).

Si on se penche du côté d'une autre forme médiatisée de BD, les blogues, il est facile de constater en consultant l'annuaire Québec Cybercomics, par exemple, que ceux-ci sont très populaires parmi les jeunes auteurs, mais que la présence féminine n'y est pas dominante. En effet, sur un total de 80 blogues répertoriés, seuls neuf sont clairement réalisés par des auteures. Il se pourrait bien donc que cette nouvelle forme de journal n'ait pas joué le rôle que lui a parfois attribué la critique dans le décloisonnement masculin du monde de la bande dessinée.

\section{Les théoriciennes}

Ce qui nous amène à la réflexion théorique sur la bande dessinée, que les femmes pratiquent au Québec. Outre Mira Falardeau, auteure de nombreux ouvrages sur la BD, dont une Histoire de la bande dessinée an Québec (2008) et Femmes et humour (2014), parmi les jeunes auteurs, Julie Delporte a signé un essai sur la «BD-réalité » dans lequel elle analyse la pratique du blogue de 
$\mathrm{BD}^{7}$ en la mettant en relation avec l'autobiographie, le journal intime, la chicklit et la télé-réalité. Elle réfléchit surtout sur la question de la liberté que le numérique et le blogue donnerait aux auteurs qui n'auraient ainsi plus besoin d'un éditeur. Toutefois, sa conclusion plutôt amère est que « Le dispositif du blogue propose un outil de liberté d'expression et de réflexion, mais les paramètres de son exécution (immédiateté, interactivité) ainsi que l'institution qui se met en place autour du dispositif (communauté, désir de popularité) détournent cet objectif premier de liberté » (Delporte 126). Cette pratique conduirait les blogueuses d'autofiction (Pénélope Bagieu, Margaux Motin) à ne reproduire que des schémas déjà existants qui sont à leur tour destinés à se reproduire dans de nombreux clones de genre girly. Toutefois, ainsi que le mentionne en passant Delporte elle-même, les blogueuses québécoises ne semblent pas être touchées par cette mode du girly (à l'exception de Bach avec son C'est pas facile d'être une fille, publié par Mécanique Générale). Les blogues sont souvent autobiographiques, mais de nature intimiste voire poétique (Journal de Delporte), ou encore humoristique (L'Ostie d'chat d'Iris et Zviane). On y trouve aussi des thèmes inattendus et originaux : dans Le quart de millimètre, Zviane donne au lecteur un cours de composition musicale.

Il est donc intéressant et significatif que les jeunes bédéistes montréalaises réfléchissent non seulement sur leurs pratiques, mais aussi sur des tendances plus générales qui touchent une certaine production féminine de la bande dessinée.

\section{Les pratiques}

La plupart des auteures sont à la fois scénaristes et dessinatrices, au moins pour une partie de leur œuvre. De plus, les collaborations sont fréquentes : Zviane et Iris pour L'Ostie d'chat, Cathon et Iris pour La liste des choses qui existent, Arsenault et Britt pour Jane, le renard et moi. Souvent, il n'y a pas de véritable division des rôles, mais une véritable coréalisation ${ }^{8}$, ainsi que l'explique Éric Bouchard pour L'Ostie d'chat:

Si [...] c'est principalement au niveau du scénario que la « fusion » d'Iris et Zviane se produit, dans cet espace où les deux personnalités extravagantes de ces jeunes artistes trouvent un terreau fertile à leur imagination avec l'élaboration des péripéties qu'auront à vivre leurs nombreux personnages, un tout autre phénomène se produit du côté du dessin. Car ces deux auteures réalisent en alternance les courts chapitres d'une même histoire, qui suit son cours indépendamment de leurs propres individualités graphiques. (Bouchard 132)

\footnotetext{
${ }^{7}$ Julie Delporte pratique elle-même le blogue BD avec son Journal, d'abord publié en anglais. Sur les traductions en anglais des BD montréalaises (Giaufret, "Le français »).

${ }^{8}$ Voir sur ce sujet l'interview à Iris et Zviane par Saint-Jacques.
} 

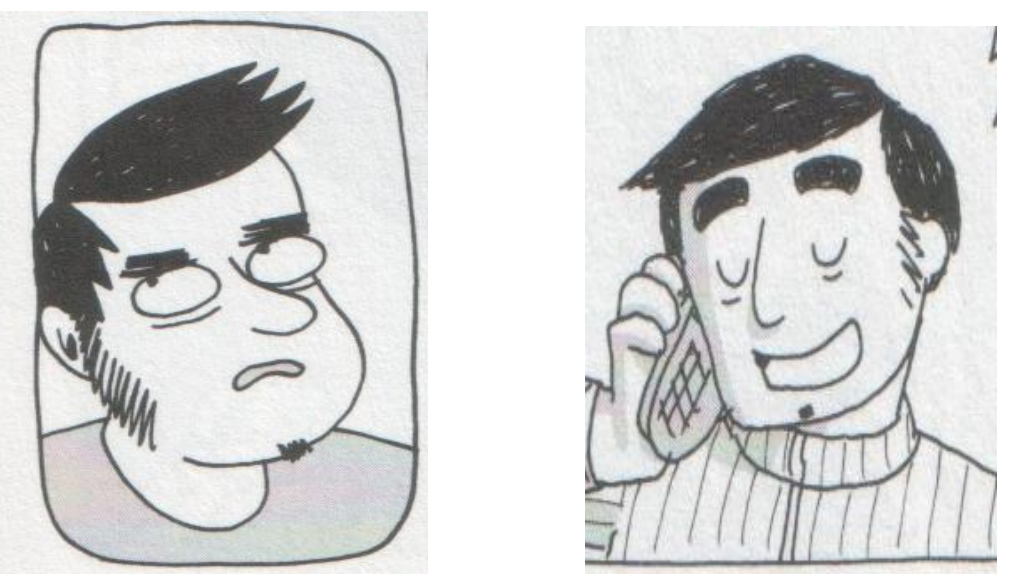

Images 1 et 2. Le personnage de Jean-Sébastien Manolli dessiné par Zviane et par Iris (L'ostie d'chat tome 35,7 ). Droits réservés.

Par ailleurs, les collaborations traversent aussi les frontières de genre : c'est le cas d'Iris et Yves Pelletier (Le pouvoir de l'amour et autres vaines romances) ou de Cathon et Alexandre Fontaine Rousseau (Les cousines vampires)

La publication avec plusieurs maisons d'édition est aussi fréquente chez les auteures, notamment les plus prolifiques: Zviane a publié chez Mécanique générale, Pow Pow, La Pastèque, Colosse, ainsi que Delcourt en France pour la version papier du blogue, réalisé avec Iris, L'Ostie d'chat. Nous allons maintenant nous concentrer de manière plus spécifique sur trois auteures : Zviane, Sophie Bédard et Sophie Yanow qui nous semblent bien incarner la diversité de la production des jeunes auteures montréalaises.

Zviane (Saint-Jacques, «Zviane »), née en 1983, est probablement la plus connue aujourd'hui parmi les jeunes auteurs montréalais, sans distinction de genre, et une des rares à être publiée en France, chez Delcourt, pour la série en trois tomes L'ostie d'chat, réalisée en collaboration avec Iris à partir d'un blogue. Son œuvre est l'image même de la diversité par les thèmes qu'elle aborde (la composition musicale, la dépression, la folie, l'amour, l'art et même les fruits), les genres (humoristique, érotique, ludique, d'introspection, l'essai en $\mathrm{BD}$ ) et les maisons d'édition (La Pastèque, Pow Pow, Mécanique Générale, Delcourt, Colosse, Graphigne, outre quelques autopublications). Le trait ainsi que l'organisation de la planche évolue et change au fil des albums, toujours en noir et blanc: des formes plus naïves du Quart de millimètre au raffinement des Deuxièmes et surtout d'Apnée (Prix Bédéis Causa 2010). 


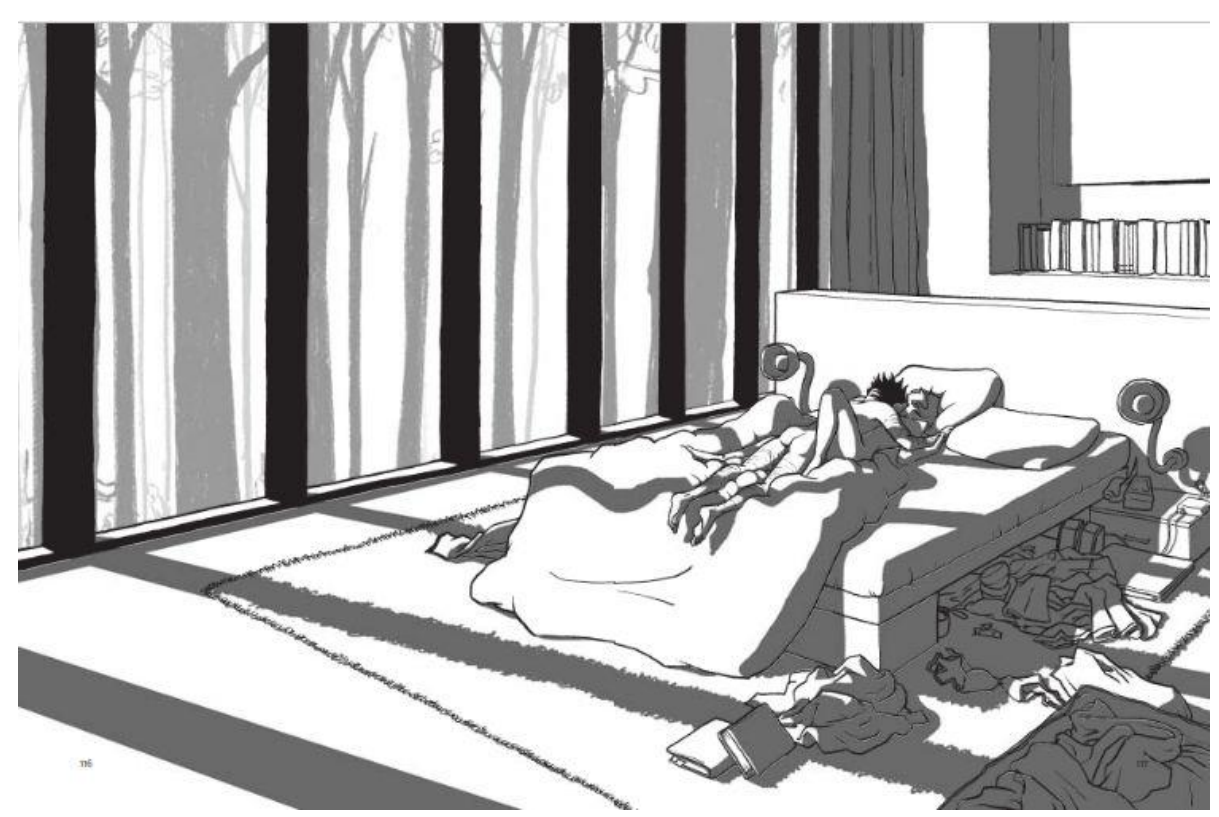

Image 3 (Zviane, Les deuxièmes 116-117). Droits réservés.

Sophie Bédard (née en 1991), collaboratrice régulière de Planches avec sa rubrique « sexologie », trace dans les quatre volumes de Glorieux printemps un portrait attachant de quatre adolescents de la banlieue montréalaise dont on suit les péripéties existentielles, amicales et amoureuses pendant leurs études au secondaire. Émilie l'intello bien rangée nulle en sport, Micheline son amie qui adore tous les excès, Mathieu le timide « invisible » et Antoine le bizarre forment un quatuor inoubliable qui interagit avec un entourage fait de professeurs, de parents pas toujours faciles à gérer et de camarades au comportement parfois incompréhensible. Fidèle à Pow Pow et à Colosse, son trait qui peut sembler naif excelle dans la représentation des relations, des émotions des adolescents qu'elle représente dans des textes extrêmement intéressants du point de vue sociolinguistique (Giaufret «Le français », « De la variation $»)$.

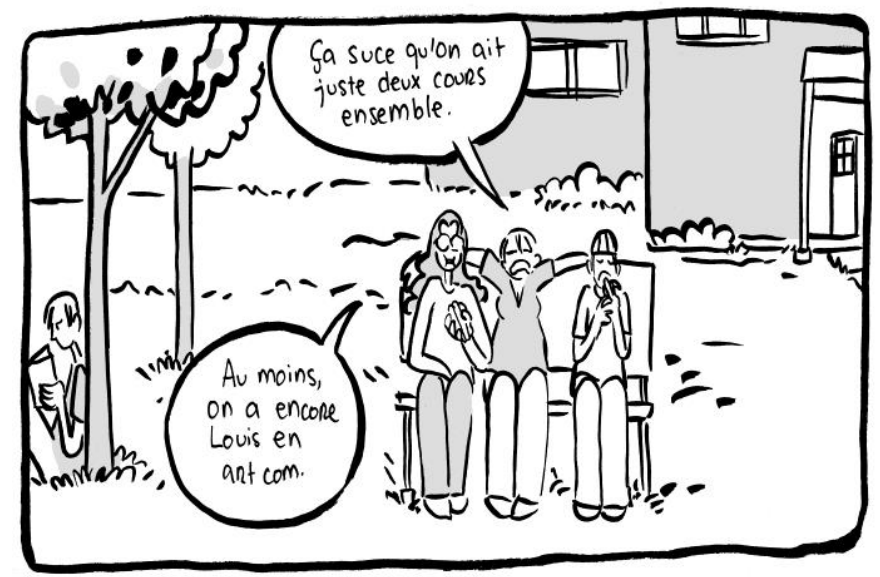

Image 4 (Bedard, Glorieux printemps tome 124$)$. Droits réservés. 
Quant à Sophie Yanow, États-Unienne de naissance, elle arrive à Montréal après un séjour à Paris. Elle y découvre le mouvement étudiant et des espaces urbains inattendus. Elle publie La guerre des rues et des maisons d'abord dans la version française — qu'elle traduit de l'anglais avec Vincent Giard - chez La mauvaise tête, puis en anglais chez Uncivilized Books (War of streets and Houses). Un quadrillage rigoureux et régulier de la planche, sans espace blanc, à quelques exceptions près, ne fait que mettre en exergue le travail sur la représentation de l'espace : cartes géographiques, plongées, contre-plongées, représentations, de la casbah d'Alger et de celle de Casablanca, du skyline et des ruelles de Montréal, des centres commerciaux, des carrefours, la géographie de Montréal et la perception de l'espace la hantent entièrement dans cette $\mathrm{BD}$, qui a reçu une nomination comme outstanding graphic novel à l'Ignatz Award 2014. Yanow représente la capacité de la scène montréalaise de la BD d'attirer de jeunes talents de l'extérieur et de leur offrir la possibilité de se faire publier et reconnaître.

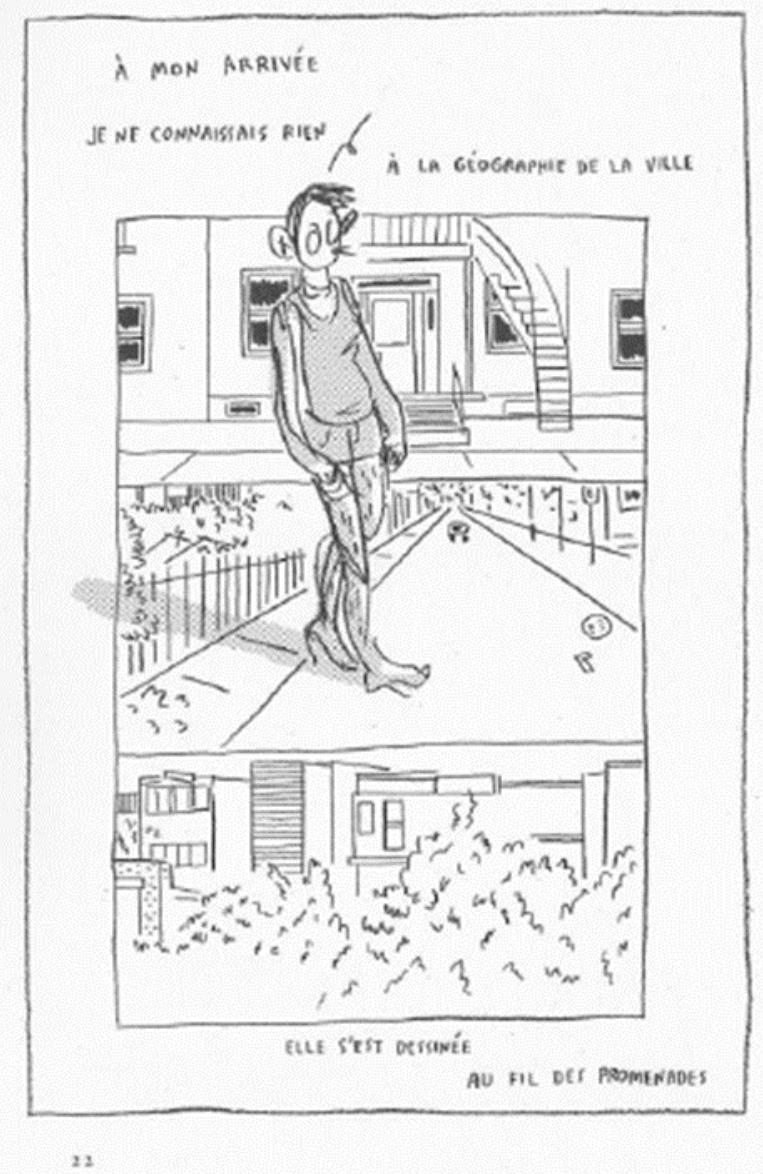

Image 5 (Yanow, La guerre des rues et des maisons 22). Droits réservés.

\section{Conclusion}


Nous espérons avoir montré la vitalité et la diversité de la bande dessinée au féminin au Québec et plus spécialement à Montréal, où de nombreuses auteures dessinent, écrivent, scénarisent et éditent des albums et des revues BD. Le pourcentage de femmes y est apparemment plus élevé que dans le milieu francophone européen, notamment chez les jeunes générations. Quant aux raisons de l'importance des femmes parmi les auteurs, nous pouvons avancer l'hypothèse qu'elles découlent d'une forte présence de maisons d'édition indépendantes, qui publient des auteurs jeunes et des romans graphiques loin des genres de la BD mainstream, notamment d'aventure, qui reste davantage masculine. Pour reprendre les propos de Marianne Saint-Jacques,

Paradoxalement, le milieu de la BDQ semble plus favorable à l'émergence d'une bande dessinée au féminin que celui de la $\mathrm{BD}$ franco-belge. [...] le marché de la $\mathrm{BD}$ québécoise, souvent fragilisé en raison de sa taille restreinte, est également beaucoup plus flexible, ce qui lui permet de réduire plus facilement certains écarts persistants, dont celui de l'espace accordé aux femmes dans le milieu du Neuvième art. (87).

La qualité n'est pas en reste : nous n'avons mentionné très rapidement que le travail de trois auteures, il en reste beaucoup d'autres dont l'œuvre est digne d'attention et riche de promesses. La jeune revue Planches pourra d'ailleurs révéler au public de nouveaux talents qui seront, nous le souhaitons, soutenus per les éditeurs, les lecteurs et les instances culturelles. 


\section{Bibliographie}

Arsenault, Isabelle, Fanny Britt. Jane, le renard et moi. Montréal : La Pastèque, 2012.

Bedard, Sophie. Glorieux printemps (tome 1). Montréal : Pow Pow, 2012. . Glorieux printemps (tome 2). Montréal : Pow Pow, 2012. . Glorieux printemps, (tome 3). Montréal : Pow Pow, 2013. Glorieux printemps, (tome 4). Montréal : Pow Pow, 2014.

Bouchard, Éric. «L'Ostie d'chat ou la BD en colocation. » Trip. 7 (2012) : $127-134$.

Cathon et Iris La liste des choses qui existent. Montréal : La Pastèque, 2013.

Cathon et Alexandre Fontaine-Rousseau. Les cousines vampires. Montréal : Pow Pow, 2014.

Delporte, Julie. La bédé-réalité. La bande dessinée autobiographique à l'heure des technologies numériques. Montréal : Colosse, 2011. . Journal. Toronto : Koyama Press, 2013. . Journal. Paris : L'Agrume, 2014.

Falardeau, Mira. Histoire de la bande dessinée au Québec. Montréal : VLB, 2008. . Femmes et humour. Sainte-Foy : Presses de l'Université Laval, 2014.

Giaufret, Anna. «Le français dans la bande dessinée québécoise : quelles représentations du français parlé? ». Repères DoRiF 2 (2013). Web. 2 aout 2015.

. «De la variation dans les bulles : le français de la bande dessinée québécoise et les dictionnaires en ligne, » In Farina, Annick, Zotti, Valeria (eds). La variation lexicale des français. Dictionnaires, bases de données, corpus. Hommage à Claude Poirier. Paris : Honoré Champion, 2014. 229-248. . «Bande dessinée et traduction à Montréal : des réseaux et des œuvres, » In Londei, Danielle, Poli, Sergio, Giaufret, Anna, Rossi, Micaela (eds) Metamorfosi della tradurione in ambito francese italiano.

Genova: Genova University Press, 2015. 147-162.

Iris, Yves Pelletier. Le pouvoir de l'amour et autres vaines romances. Montréal : La Pastèque, 2014.

Iris et Zviane. L'ostie d'chat, (tome 1). Paris : Delcourt, 2011. . L'ostie d'chat (tome 2). Paris : Delcourt, 2012. . L'ostie d'chat (tome 3). Paris : Delcourt, 2012.

Rannou, Maël. «Planches et Sentinelle ». du9. L'autre bande dessinée (2015). Web. 29 juillet 2015.

Ratier, Gilles. «2014, l'année des contradictions. Rapport sur la production d'une année de bande dessinée dans l'espace francophone européen. » 2014. Web. 2 aout 2015.

Saint-Jacques, Marianne. «Iris et Zviane (L'ostie d'chat) : « Notre but premier dans ce projet, c'est d'avoir du fun. »» ActuaBD. 2011. Web. 29 juillet 2015. . «Zviane, nouvelle voix de la BD québécoise. » ActuaBD. 2011. Web. 29 juillet 2015 . 
. «BDQ : cherchez la femme ». Sentinelle. 2015 : 76-87

Viau, Michel. «BDQ 2014 : l'année de la diversité. » 2014. Web. 2 aout 2015. . Histoire de la bande dessinée an Québec (tome 1). Montréal : Éditions Mém9ire, 2014.

Yanow, Sophie. La guerre des rues et des maisons. Montréal : La mauvaise tête, 2013.

War of Streets and Houses. Minneapolis : Uncivilized Books, 2014.

Zviane. Les deuxièmes. Montréal : Pow Pow, 2013. - Apnée. Montréal, Pow Pow, 2010. Le bestiaire des fruits. Montréal : La Pastèque, 2014.

. Pain de viande avec dissonances. Montréal : Pow Pow, 2011. . Ping Pong. Montréal : Pow Pow, 2015.

\section{Sitographie}

Bach [Estelle Bachelard]. Bach. Illustrations + BD. Web.4 août 2015. BDQC. ARTV. Web. 4 août 2015.

Bédard, Sophie. Glorieux printemps. Web. 4 août 2015.

Collectif des créatrices de bande dessinée contre le sexisme. Web. 4 août 2015.

Colosse. Web. 4 août 2015.

Delporte, Julie. Le dernier kilomètre. Web. 4 aoû.t 2015.

Drawn \& Quarterly. Web. 4 août 2015

Magnette, Christophe, Delporte, Julie, Boutin, Laurent. Dans ta bulle.

CHOC.ca. Web. 4 août 2015.

La Maison de la bande dessinée de Montréal. Web. 4 août 2015

La Mauvaise tête. Web. 4 août 2015.

Mécanique générale. Web. 4 août 2015.

L'Oie de Cravan. Web. 4 août 2015.

Paradis, Rémi, Bernier, Mathieu. Québec Cybercomics. Web. 4 août 2015.

La Pastèque. Web. 4 août 2015.

Planches. Revue de bandes dessinées d'auteurs Québécois. Web. 4 août 2015.

Pow Pow. Web. 4 août 2015.

Yanow, Sophie. Sophie Yanow. Web. Web. 4 août 2015.

Zviane [Sylvie-Anne Ménard]. Le blogue de Zviane. Web. 4 août 2015. 